\title{
Estudio morfométrico de las fibras nerviosas del nervio oculomotor del perro
}

\author{
Morphometric study of the nerve fibers of the oculomotor nerve in dog \\ J Vivo*, J L Morales, A Diz, A M Galisteo, J G Monterde, E Agüera, F Miró \\ Departamento de Anatomía y Anatomía Patológica Comparadas, Campus Rabanales, \\ Universidad de Córdoba, Córdoba, España.
}

\begin{abstract}
SUMMARY
The intracranial portion of the right oculomotor nerves was extracted from six adult German Shepherd dogs. The nerves were studied using light and electron microscopy. Nerve area was calculated, and myelinated and unmyelinated fibers were analized to determine number, diameter and area. Also in the myelinated fibers the area and diameter of the correspondent axon, and thickness of the myeline sheath were measured. The mean number of fibers was $8543.50 \pm 1231.85$ for myelinated and $1402.00 \pm 241.58$ for unmyelinated. The mean fiber diameter was $10.23 \pm 0.68 \mu \mathrm{m}$ for myelinated and $0.43 \pm 0.21 \mu \mathrm{m}$ for unmyelinated. This study demonstrated that the oculomotor nerves in dogs have structural and ultrastructural characteristics that are similar to those from other species.
\end{abstract}

Palabras clave: nervio oculomotor, fibras mielínicas, amielínicas, perro.

Key words: oculomotor nerve, myelinated, unmyelinated fibers, dog.

\section{INTRODUCCION}

El nervio oculomotor (N. oculomotorius), de todas las especies, está constituido en su origen por fibras nerviosas mielínicas que proceden del núcleo motor del nervio oculomotor, así como por las fibras parasimpáticas preganglionares que se incorporan desde el núcleo parasimpático del nervio oculomotor, ambos situados en el tronco del encéfalo (Brodal 1981). Este nervio es el encargado de llevar impulso nervioso a los músculos extraoculares recto dorsal, medial y ventral, oblicuo dorsal (Vivo y col 2005) y al elevador del párpado, así como a los músculos lisos ciliar y esfínter de la pupila, a cuyo fin le vemos emerger por la cisura orbitaria. No obstante, en este nervio se han encontrado, en diferentes especies, distintos tipos de fibras, tanto en base a sus características morfológicas -mielínicas y amielínicas - como a su velocidad de conducción (Manni y col 1987, 1989). Las características fibrilares de este nervio han sido analizadas en diversas especies. Algunos estudios se han centrado en examinar el significado funcional de estas fibras en la oveja (Manni y col 1978, 1987, 1989; Bortolami y col 1977), en el gato (Porter y Spencer 1982) y hombre (Lanzino y col 1993, Hayakawa y col 2000). Otras investigaciones han considerado algunas de sus características morfométricas fibrilares, igualmente en una gran variedad de especies: en la rata (Fraher 1989a, 1989b), oveja (Berardinelli y

Aceptado: 11.07.2006.

E-mail: an1viroj@uco.es Fax: int +34 957218682. Teléfono: int +34957218675 col 2000), gato (Batini y col 1979, Hildebrand y col 1988) y el hombre (Kobayashi y col 1998, Kawamura y col 2000). En el perro las únicas referencias encontradas en la literatura respecto a los nervios de los músculos extraoculares se refieren al nervio oculomotor (Bortolami y col 1977) y al nervio troclear (Vivo y col 2004a, 2004b). Por lo tanto, son muy pocos los estudios mencionados en la literatura que hayan realizado un análisis en detalle de las características morfológicas y morfométricas de las fibras nerviosas del nervio oculomotor del perro.

Nuestro objetivo es determinar en el perro el área del nervio oculomotor, y sobre las fibras mielínicas y amielínicas analizar su número, diámetro y área, así como el área y el diámetro del axón correspondiente y el grosor de la vaina de mielina, de las fibras mielínicas.

\section{MATERIAL Y METODOS}

Para este estudio se han utilizado seis perros sanos adultos, entre 4 y 6 años de edad, de raza pastor alemán de entre $40-50 \mathrm{~kg}$ de peso, obtenidos del lazareto municipal donde fueron eutanasiados por sobredosis anestésica con tiopental (Tiobarbital ${ }^{\circledR}$ ). Tras su sacrificio se ha levantado la bóveda del cráneo y obtenido el nervio oculomotor, derecho en su recorrido intracraneal.

Los nervios se fijaron en una solución al $2 \%$ de glutaraldehído preparado en un buffer fosfato $0,1 \mathrm{M}$ (pH 7,4), durante la noche se mantuvieron a $4^{\circ} \mathrm{C}$ y fueron fijados durante 30 minutos en una solución de tetróxido de osmio al $1 \%$ preparado en un buffer fosfato $0,1 \mathrm{M}(\mathrm{pH} 7,4)$. Tras deshidratar, en gradientes de etanol, 
fueron incluidos en araldita y cortados con un ultramicrotomo LKB en secciones transversales semifinas $(400 \mathrm{~nm})$ y ultrafinas $(70 \mathrm{~nm})$. Las secciones semifinas fueron teñidas con azul de toluidina y utilizadas para su examen con microscopio óptico, mientras que las secciones ultrafinas fueron doblemente teñidas con acetato de uracilo y citrato de plomo. Las secciones ultrafinas se estudiaron y fueron fotografiadas en un microscopio electrónico de transmisión Philips CM10.

Las secciones semifinas transversas de los nervios enteros fueron digitalizadas en sucesivas imágenes a 40x, con las que se reconstruyó completamente la sección nerviosa obtenida; por su parte, las secciones ultrafinas fueron examinadas a 10500x y fotografiadas a 25000x. Con el software Sci Image (1998 Scion corporation 82 Worman's Mill Court Suite H Frederick, Maryland 21703) se evaluaron en las imágenes semifinas digitalizadas el área, diámetro mayor y diámetro menor del $25 \%$ de las fibras nerviosas mielínicas y de sus axones correspondientes; por su parte, sobre la reconstrucción completa del nervio se ha medido su área y se ha contado el número de todas las fibras nerviosas mielínicas. Sobre las secciones ultrafinas se seleccionaron cuadrados, elegidos al azar, equivalentes a una superficie del $25 \%$ del nervio entero (Berardinelli y col 2000), en los que se ha contado el número de fibras nerviosas amielínicas y sobre las que se han evaluado su área, diámetro mayor y diámetro menor. Todas las mediciones fueron realizadas por el mismo operador. Conociendo previamente la superficie completa del nervio, el número total de fibras amielínicas de cada nervio se calculó por una simple regla aritmética. Los datos se trataron con el programa Microsoft Office Excel 2003a, con el que se obtuvo, de cada nervio, para las fibras mielínicas y amielínicas, el porcentaje de cada tipo de fibras respecto al total del nervio, el diámetro medio de la fibra nerviosa, su densidad (número de fibras por $\mathrm{mm}^{2}$ ), área media, área total (suma del área de todas las fibras) y área relativa (expresada en porcentaje del área del nervio). Además, sobre las fibras mielínicas se calcularon, el área media del axón y su diámetro medio, el grosor de la vaina de mielina ((diámetro fibrilar menos diámetro del axón)/2) y la razón diámetro axón/ diámetro fibrilar. Para cada nervio y para las variables que procediera se obtuvo la desviación estándar (DE intra). Fueron calculadas la media global (obtenida a partir de la media de cada nervio) y su correspondiente desviación estándar (DE inter) y la media de la estándar intra.

\section{RESULTADOS}

FIBRAS MIELINICAS. Las fibras nerviosas mielínicas fueron identificadas al microscopio óptico en todos los nervios examinados gracias a las características de sus vainas de mielina.
En la sección transversal de los nervios se observó, al microscopio óptico, una gran variedad en los tamaños de las fibras mielínicas (figura 1). El estudio de frecuencias mediante histogramas de los datos globales para cada nervio (todos los datos de los seis nervios analizados) mostró que la variable fibrilar grosor de la vaina de mielina presentaba una distribución bimodal (figura 2). No se encontró distribución bimodal para el resto de variables de este nervio. En cualquier caso, las fibras, independientemente de su tamaño, se distribuían en el nervio de forma homogénea, no detectándose zonas donde predominara alguna de ellas.

El área media de los nervios oculomotores fue de $1.155 .145,70 \pm 193.007,14{\mu \mathrm{m}^{2}}^{2}$. El número medio de fibras mielinizadas presentes en los nervios fue de 8.543 (rango 7.785-10.373) que representan el 85,90 $\pm 2,00 \%$, del total de las fibras del nervio y que ocupan el 69,81\% de la superficie total del nervio. El diámetro fibrilar medio es 10,23 $\mu \mathrm{m}$ (rango 2,10-23,82), siendo el intervalo donde se presentan un mayor número de fibras de 12 a 13,5 $\mu \mathrm{m}$ (figura 2). El diámetro del axón osciló entre 1,05-14,79 (media 6,22 $\mu \mathrm{m}$ ). En cuanto al grosor de la vaina de mielina presentó un rango de 0,35-5 (media 2,01 $\mu \mathrm{m}$ ). Dada la distribución bimodal de esta variable en el nervio oculomotor, el intervalo de separación de las dos poblaciones fibrilares fue de 1,5-1,75 $\mu \mathrm{m}$.

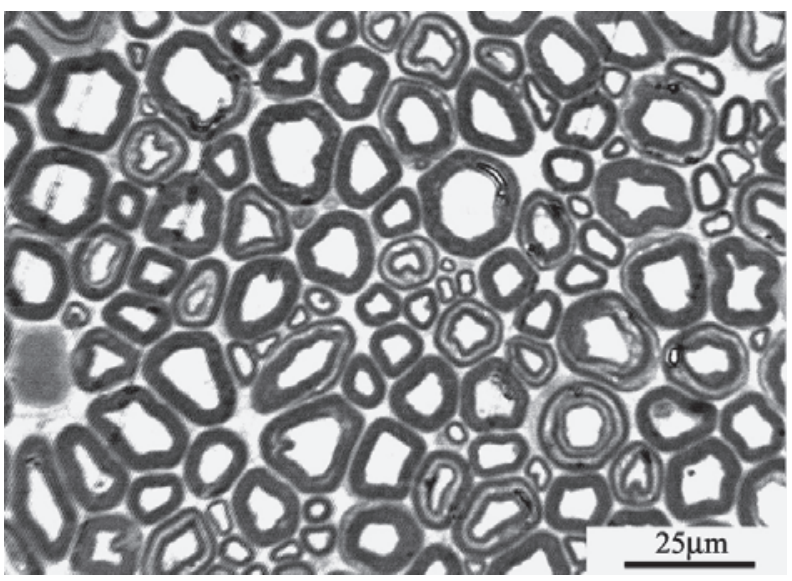

Figura 1. Micrografía óptica de un corte transversal del nervio oculomotor, observándose las característica morfológicas de las fibras mielínicas.

Light micrograph of transverse sections of the oculomotor nerve in dog showing the morphologic characteristics of myelinated fibers.

FIBRAS AMIELINICAS. Las fibras amielínicas se encontraron en todos los nervios entre las fibras mielínicas, con morfología redondeada u oval, englobadas por las células de Schwann en grupos de 2-3 fibras nerviosas; no obstante, también se observaron células de Schwann con un número mayor de fibras amielínicas en todos los nervios estudiados (figura 3). Los histogramas de frecuencias de los datos globales de cada nervio mostraron 

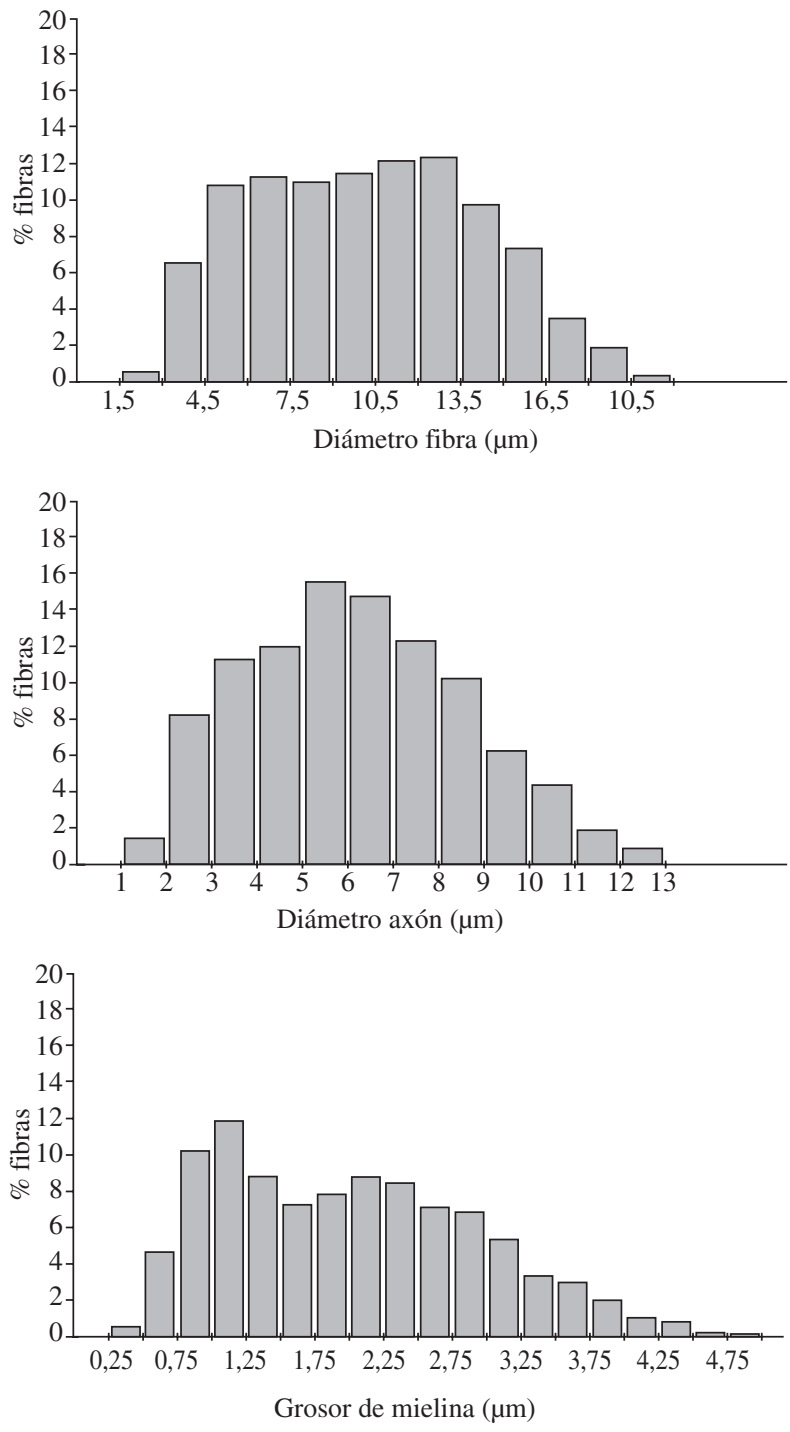

Figura 2. Histogramas de las fibras mielínicas del nervio oculomotor del perro.

in dogs.

Histograms of myelinated fibers of the oculomotor nerve

que las variables fibrilares (área y diámetro) presentaban una distribución unimodal.

El número medio de fibras amielínicas presentes en los nervios estudiados fue de 1.402 (rango 1.062-1.543) que representan el $14,10 \pm 2,00 \%$ del número total de las fibras del nervio y ocupan el $0,02 \%$ de la superficie total del nervio.

El diámetro fibrilar osciló entre 0,11-2,1 (media $0,43 \mu \mathrm{m})$, siendo el intervalo donde se concentra el mayor número de fibras de 0,2-0,3 $\mu \mathrm{m}$ (figura 4).

Los valores medios y la variabilidad (DE intra medio y DE inter) de cada una de las variables tanto para las fibras mielínicas como amielínicas se exponen en el cuadro 1.
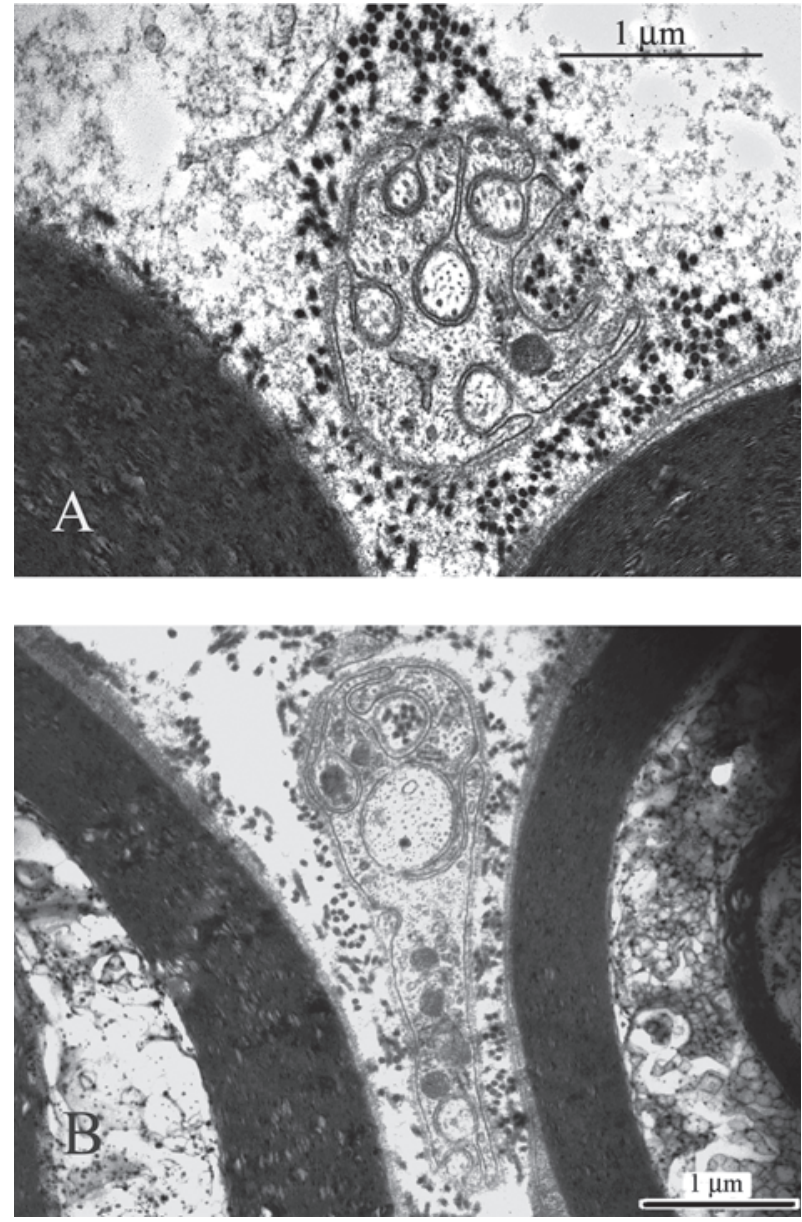

Figura 3. Micrografías electrónicas de cortes transversales del nervio oculomotor del perro, observándose fibras mielínicas y amielínicas (A: x 25000, B: x 19000).

Electron micrographs of transverse sections of the oculomotor nerve in dogs showing myelinated and unmyelinated fibers (A: x 25000, B: x 19000).

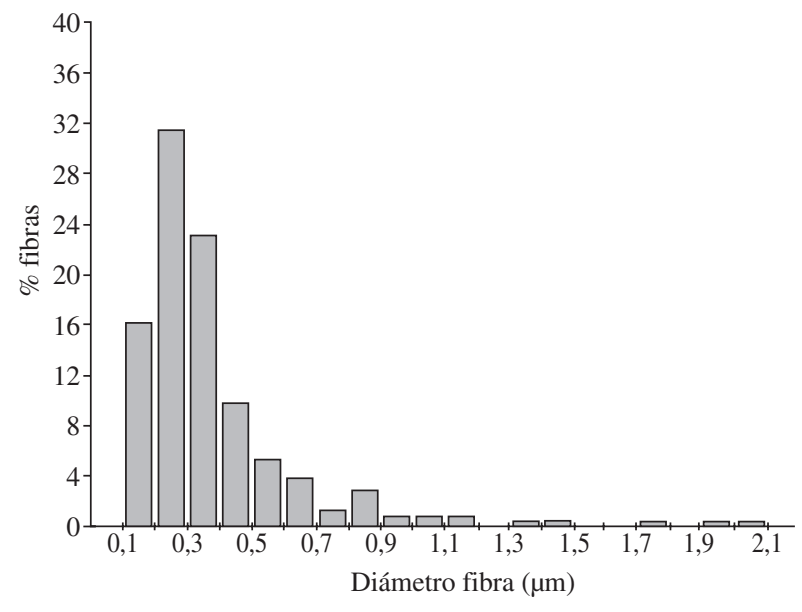

Figura 4. Histograma de las fibras amielínicas del nervio oculomotor del perro.

Histograms of unmyelinated fiber diameters of the oculomotor nerve in dogs. 
Cuadro 1. Medias y desviaciones típicas de cada variable analizada de las fibras mielínicas y amielínicas. Means and standard deviations of the myelinated and unmyelinated fibers of each analyzed variable.

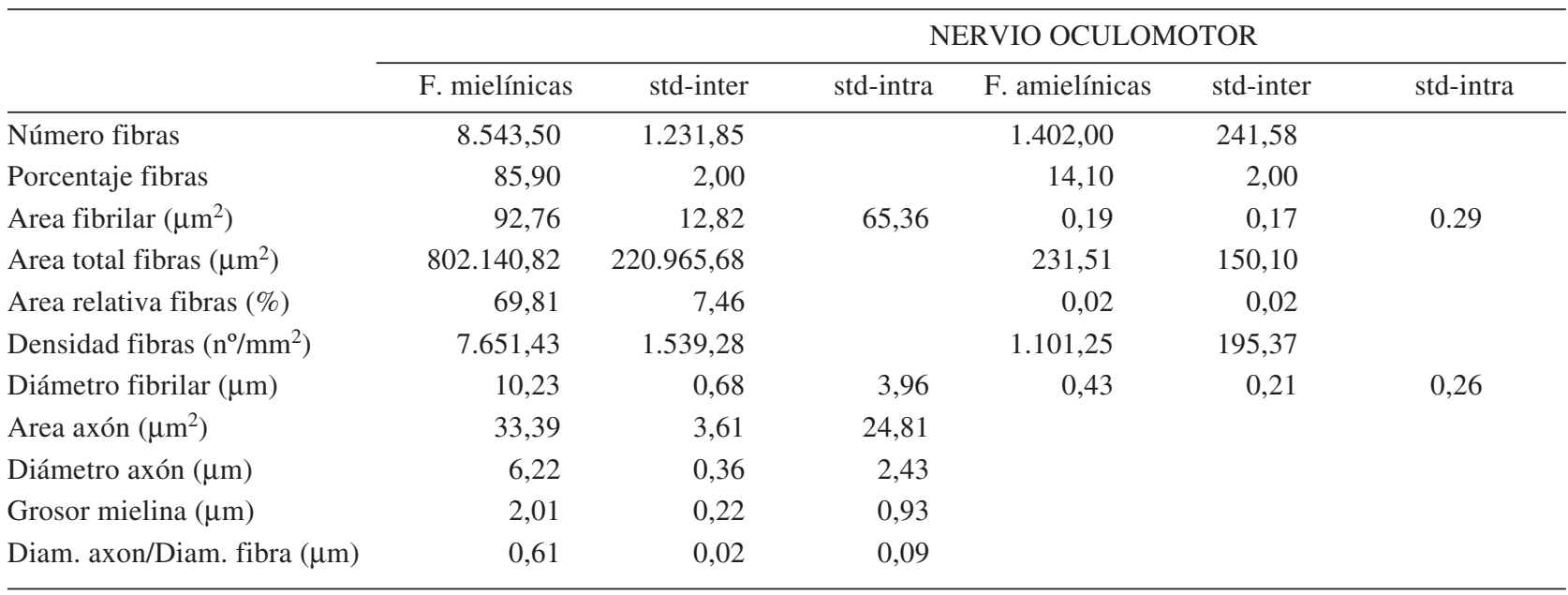

\section{DISCUSION}

Sobre el nervio oculomotor (III) existe una abundante literatura en determinadas especies, prestándose una menor atención al perro, y especialmente a los aspectos morfométricos de sus fibras nerviosas mielínicas y amielínicas. Las únicas referencias encontradas en la literatura respecto a los nervios de los músculos extraoculares, en esta especie, se refieren al nervio oculomotor (Bortolami y col 1977) y al nervio troclear (Vivo y col 2004a, 2004b). Por lo tanto, son muy pocos los estudios que hayan realizado un análisis en detalle de las características morfológicas y morfométricas de las fibras nerviosas del nervio oculomotor del perro.

El espectro de tamaño de las fibras nerviosas del nervio oculomotor del perro, obtenido en nuestro estudio, es similar al determinado en la oveja (Berardinelli y col 2000), algo superior al señalado en el gato (Batini y col 1979) y mucho más amplio al determinado en el mono, para el ramo del nervio oculomotor destinado al músculo oblicuo ventral (Ruskell 1983). Este espectro de tamaño presenta una distribución unimodal, lo que difiere de lo apuntado en el gato (Batini y col 1979), en la oveja (Berardinelli y col 2000) y en el mono (Ruskell 1983).

El número de fibras mielínicas halladas en nuestro estudio para el nervio oculomotor es menor que en la oveja (Berardinelli y col 2000) y mayor que en la rata (Fraher 1989a) y el gato (Batini y col 1979); no obstante, los porcentajes fibrilares obtenidos en nuestra experiencia son muy similares a los encontrados en la oveja (Berardinelli y col 2000) y menores a los señalados en la rata (Fraher, 1989a). El número de fibras nerviosas es proporcional al número de músculos (Batini y col 1979), y más concretamente al número de fibras musculares a los que inerva cada nervio. No obstante, es de presupo- ner que no todas las fibras mielínicas de estos nervios sean exclusivamente motoras, pues los estudios realizados en la oveja y en el hombre (Abo-El-Enene 1978) señalan que un importante número de las fibras mielínicas del nervio oculomotor son fibras aferentes propioceptivas de grande y de pequeño tamaño.

La relación diámetro axón/diámetro fibra que hemos determinado en el nervio oculomotor del perro es sensiblemente inferior al determinado en el gato (Fraher 1989b), en la rata (Fahrenkamp y Friede 1987) y para el ramo medial del nervio oculomotor de la rata y el hombre (Bardosi y col 1990), lo que nos señala que el espesor de las vainas de mielina es proporcionalmente mayor, para este nervio y a los niveles indicados, en el perro que en la rata, gato y hombre.

En todos los nervios analizados hemos observado, al igual que en la rata (Fraher 1989a) y en la oveja (Berardinelli y col 2000), las fibras amielínicas distribuidas en grupos por todas las partes de la sección transversal del nervio. El número de fibras amielínicas determinado en esta experiencia, para el nervio oculomotor, es sensiblemente inferior al señalado en el hombre (Kawamura y col 2000). Estas fibras amielínicas representan un porcentaje considerable del total de las fibras nerviosas, siendo del 14,10\% en el nervio oculomotor. Este porcentaje es muy similar a los determinados en la oveja (Berardinelli y col 2000) y a los señalados en la rata (Fraher 1989a), siendo superior al presentado en el mono (Ruskell 1983), para el ramo del nervio oculomotor que inerva al músculo oblicuo ventral. Respecto al área transversa de las fibras amielínicas, los datos determinados en este estudio son muy inferiores a los aportados en el hombre (Kawamura y col 2000).

Según Bortolami y col (1977) las fibras amielínicas del nervio oculomotor tienen su cuerpo neuronal en el 
ganglio semilunar y recogen sensibilidad de los territorios oculares por la rama oftálmica del trigémino terminando estas aferencias mayoritariamente en el subnúcleo gelatinoso del núcleo caudal del trigémino.

En conclusión, nuestro estudio sobre el nervio oculomotor muestra que este nervio, en el perro, presenta características morfométricas, estructurales y ultraestructurales semejantes a las de otras especies.

\section{RESUMEN}

En este estudio se utilizaron seis perros adultos, de raza pastor alemán, de los que fueron extraídos los nervios oculomotores derechos en su porción intracraneal. Los nervios se analizaron con microscopia óptica y microscopia electrónica. En todos los nervios se calculó su área y analizaron las fibras mielínicas y amielínicas. En ambos tipos de fibras se determinó su número, diámetro y área. En las fibras mielínicas, además, se determinó el área y el diámetro del axón correspondiente y el grosor de la vaina de mielina. El número medio de fibras fue de $8.543,50 \pm 1.231,85$ mielínicas y $1.402 \pm 241,58$ amielínicas. Los diámetros medios fibrilares fueron de $10,23 \pm 0,68 \mu \mathrm{m}$ en las fibras mielínicas y de 0,43 $\pm 0,21 \mu \mathrm{m}$ para las amielínicas. Nuestro estudio sobre los nervios oculomotores muestra que estos nervios, en el perro, presentan características morfométricas, estructurales y ultraestructurales semejantes a las de otras especies.

\section{REFERENCIAS}

Abo-El-Enene MA. 1978. Propioceptive afferent fibers in the cranial nerves III, IV and VI. Acta Anat 101, 62-65.

Bardosi A, J Sallo, C Schafer, H Muhlendy. 1990. Morphometric comparison between human and rat abducens and oculomotor nerves. Acta Anat 138, 24-31.

Batini C, C Buisseret-Delmas, RT Kado. 1979. On the fibers of the III, IV and VI cranial nerves of the cat. Arch Ital Biol 117, 111122.

Berardinelli P, PA Scapolo, AM Barazzoni, V Russo, R Bortolami. 2000. Never fiber composition of the intracranial portion of the oculomotor, trochlear, and abducens nerves in the sheep. Anat Rec 260, 294-298.

Bortolami R, A Veggetti, E Callegari, ML Lucchi, G Palmieri. 1977. Afferent fibers and sensory ganglion cells within the oculomotor nerve in some mammals and man. I. Anatomical investigations. Arch Ital Biol 115, 355-386.

Brodal A. 1981. Neurological anatomy in relation to clinical medicine. Oxford University Press.
Fahrenkamp I, RL Friede. 1987. Characteristic variations of relative myelin sheath thickness in 11 nerves of the rat. Anat Embryol $177,115-21$.

Fraher JP. 1989a. Axon numbers in rat oculomotor, trochlear and abducent nerves. J Anat 166, 151-155.

Fraher JP. 1989b. Axon-myelin relationships in rat cranial nerves III, IV, and VI: a morphometric study of large - and small -fibre classes. J Comp Neurol 15, 384-390.

Hayakawa T, M Itoh, T Miki, T Kaneto, H Tomiyama, Y Takeuchi. 2000. Sympathetic fiber innervating the extraocular muscles: cells of origin in the cat superior cervical ganglion. Okajimas Folia Anat Jpn 77, 119-124.

Hildebrand C, A Oscarsson, M Risling. 1988. Fiber composition of the feline trochlear and abducens nerves. Brain Res 453, 401-407.

Kawamura N, K Matsumoto, A Wada, N Goto. 2000. Unmyelinated nerve fiber analysis of the human oculomotor nerve. Okajimas Folia Anat Jpn 77, 59-62.

Kobayashi N, Y Sawabe, K Matsumoto, N Otsuka, N Goto. 1998. Unmyelinated nerve fiber analysis of the human abducent nerve. Okajimas Folia Anat Jpn 74, 329-35.

Lanzino G, A Andreoli, F Tognetti, P Limoni, F Calbucci, R Bortolami, ML Lucchi, E Callegari, C Testa. 1993. Orbital pain and unruptured carotid-posterior communicating artery aneurysms: the role of sensory fibers of the third cranial nerve. Acta Neurochir (Wien) 120, 7-11.

Manni E, R Bortolami, VE Pettorossi, ML Lucchi, E Callegari. 1978. Afferent fibers and sensory ganglion cells within the oculomotor nerve in some mammals and man. II. Electrophysiological investigations. Arch Ital Biol 116, 16-24.

Manni E, R Bortolami, VE Pettorossi, ML Lucchi, E Callegari, F Draicchio. 1987. Influence of oculomotor nerve afferents on central endings of primary trigeminal fibers. Arch Ital Biol 126, 29-39.

Manni E, F Draicchio, VE Pettorossi, C Carobi, S Grassi, R Bortolami, ML Lucchi. 1989. On the nature of the afferent fibers of oculomotor nerve. Arch Ital Biol 127, 99-108.

Porter JD, RF Spencer. 1982. Localization of morphology of cat extraocular muscle afferent neurones identified by retrograde transport of horseradish peroxidase. J Comp Neurol 204, 56-64.

Ruskell GL. 1983. Fibre analysis of the nerve to the inferior oblique muscle in monkeys. J Anat 137, 445-455.

Vivo J, JL Morales, A Diz, AM Galisteo, JG Monterde, A Blanco, E Agüera. 2004. Intracranial portion of the nerve and dorsal oblique muscle composition in dog: a structural and ultrastructural study. J Morphol 262, 708-713.

Vivo J, JL Morales, A Diz, A Blanco, JG Monterde, F Miró. 2004. Estudio estructural y ultraestructural de la porción intracraneal del nervio troclear del perro. An Vet 20, 105-111.

Vivo J, JL Morales, A Diz, F Miró, AM Galisteo, E Agüera. 2005. Estudio morfológico y morfométrico del músculo oblicuo dorsal del perro. Arch Med Vet 37, 49-54. 